\title{
EMPODERAMIENTO COMUNITARIO COMO EJE TRANSFORMADOR EN LOS PROCESOS DE INNOVACIÓN SOCIAL
}

\section{Entorno Ambiente}

Katherine Elizabeth López Pabón

kaatheelopez06@gmail.com

Jessith Fernando Guerrero Escobar

jfg.caos@gmail.com

Universidad de Nariño.

Pasto, Nariño.

Co-diseño, co-creación, empoderamiento comunitario,

Palabras Clave: estrategias metodológicas, innovación social, laboratorios ciudadanos, valor público. , 


\section{Resumen}

El artículo hace parte de un proceso que, más allá de un ejercicio académico, quiere aportar

Ldesde la investigación-creación, con una estrategia metodológica para el desarrollo de procesos de innovación social desde y para las comunidades rurales del Departamento de Nariño. Con una población mayoritariamente rural, Nariño, históricamente, ha sufrido problemáticas ambientales y sociales; pero, cuenta con potencialidades como la biodiversidad, su gente y cultura, entre otras características que pueden ser fortalecidas mediante estrategias de innovación social en el contexto rural. Por tanto, es necesario proyectarse al cambio mediante nuevos procedimientos y métodos que busquen respuestas alternativas a problemáticas comunes, al permitir la interacción entre las personas y agentes externos con capacidades y habilidades técnicas, que procuren el empoderamiento comunitario y su valor público, mediante el co-diseño, la experimentación y la implementación de prototipos, para estimular la colaboración y brindar nuevas habilidades que ofrezcan mayores posibilidades para el desarrollo local, en articulación directa entre la comunidad, la academia y las instituciones. La innovación social para el desarrollo integral y cultural de las comunidades rurales, surge a partir del empoderamiento comunitario; para ello es indispensable desarrollar estrategias metodológicas basadas en el concepto de laboratorios ciudadanos, aplicados mediante manuales de experiencias tipo kit de herramientas, que permitan acceder al conocimiento y, así, generar, desde las habilidades personales, alternativas a dichas problemáticas, teniendo en cuenta los liderazgos y sus acciones enfocados en la transformación comunitaria.

\section{Introducción}

“En 2017 aumentó el abismo entre millonarios y pobres”, fue el título del artículo del periódico El Tiempo del 27 de enero de 2018, firmado por Armando Neira. Allí se expone cómo en el Foro Económico de Davos se dio a conocer por parte de Oxfam el informe sobre desigualdad, cuyo propósito era hacer campaña por un futuro más justo. En el artículo se resalta que:

El 82 por ciento de la riqueza mundial generada durante 2017 fue a los bolsillos del 1 por ciento más rico de la población, mientras el 50 por ciento más pobre -3.700 millones de personas- no recibió nada de dicho crecimiento.

De acuerdo a lo anterior, también es pertinente citar a Barroso (2011), quien menciona: "La crisis financiera y económica hace que la creatividad y la innovación en general y la innovación social en particular sean aún más importantes para fomentar el crecimiento sostenible, empleo seguro e impulsar la competitividad" (p.1).

En el contexto nacional, según el informe sociodemográfico que hace el Departamento Nacional de Planeación (DNP, 2015), Nariño tiene un 53\% de población mayoritariamente rural y es, por tanto, fundamentalmente agropecuario. Históricamente ha sufrido un aislamiento geopolítico que ha conllevado a muchas problemáticas sociales y económicas, 
como la pérdida del relevo generacional en el campo, de saberes ancestrales, el abandono de la agricultura familiar, el deterioro de la cultura y la autonomía campesina. Tales factores son generadores, entre otros, de la pobreza rural que inhibe el desarrollo óptimo del sector agrario. Además, ha sido un departamento azotado por la violencia y las prácticas ilícitas que han generado desigualdad e inequidad en las comunidades y, sobre todo, le han quitado valor y dignidad al campesino, impidiendo la transferencia de conocimiento y limitando la divulgación de los procesos que se llevan a cabo en el territorio.

Por consiguiente, se han debilitado las capacidades de las personas para afrontar sus propias problemáticas, llevándolas a una práctica sociocultural conformista, apática y cómoda, en dondelaúnica manera desobrellevar dichas problemáticas hasido medianteelasistencialismo estatal, institucional y politiquero, rodeado de un halo de corrupción constante. Estas prácticas sólo se han centrado en el ejercicio de la ejecución de programas asistencialistas, sin tener en cuenta una real participación de las comunidades en la gobernanza territorial que les permita incidir en la formulación, desarrollo y ejecución de programas integrales para garantizar sus derechos y una vida digna. Sin embargo, y frente a esas problemáticas, Nariño tiene muchas potencialidades para superarlas. Cuenta con una situación geopolítica privilegiada, posee riquezas naturales, lo que lo hace un departamento altamente biodiverso. Esencialmente cuenta con una población caracterizada por su gran ingenio, laboriosidad y, sobre todo, por un amplio sentido de solidaridad y cooperación social.

Velásquez (2003) menciona que la participación de la comunidad se convierte en un medio para la formulación, discusión y adopción de los grandes consensos sobre el desarrollo y la convivencia en el territorio, lo que equivale a un ejercicio de empoderamiento de una comunidad en la toma de decisiones para lograr una transformación efectiva y significativa en su diario vivir.

Para el desarrollo del proceso se ha tenido en cuenta datos tales como el Índice de Necesidades Básicas insatisfechas (INB), extraído del Plan de Desarrollo 2016 - 2019 del Departamento de Nariño, el cual menciona que entre 1993 y 2005 ha pasado de $56.31 \%$ a $43.79 \%$. Se nota una reducción en el porcentaje, pero aun así dicho nivel sigue siendo alto. También se puede mencionar que en el informe Plan de Energización Rural Sostenible (PERS) de 2014, para el Plan Nacional de Desarrollo 2014 - 2018, se muestra que el índice de vulnerabilidad en los municipios más rurales de Nariño es de 53,2 \%, y el índice de vulnerabilidad en los municipios menos rurales es de 51,8 \%, siendo mayor al índice promedio nacional. Por tanto, los municipios del Departamento de Nariño tienen un alto grado de vulnerabilidad que se entiende como la escasez de recursos y deficiente infraestructura, entre otros.

Para abordar este punto, se hace necesario referirse también a los distintos procesos que se han llevado a cabo en el Centro de Innovación Social de Nariño (CISNA), como el AntiHakaton, visualización analógica de problemáticas específicas que referencia poblaciones y comunidades en el territorio nariñense. Igualmente, ejercicios como el denominado Innovación Social para el Agro (SURCO), enfocado en la investigación del campo nariñense y sus características. $Y$ el proceso de construcción participativa y 
colaborativa de la Política Pública de Innovación Social de Nariño (POLISIS), desarrollada en talleres con representantes de los 64 municipios del Departamento de Nariño, en más de 20 mesas de trabajo en las 13 subregiones del mismo, con una participación de más de 1.700 personas. Estas determinaron la importancia que tiene la innovación social y lo que eso implica en el desarrollo territorial a partir del empoderamiento comunitario. En tal sentido, se estableció la implementación de estrategias que puedan dar herramientas a las comunidades para que puedan generar nuevos proyectos y alternativas de solución que mitiguen algunas problemáticas en sus contextos cotidianos.

\section{Innovación Social en el Territorio Rural}

Para Baskerville (1999) la Investigación para la Acción parece ser perfectamente adecuada para llevar a cabo una investigación conjunta, la experimentación, la evaluación y las actividades de validación en un laboratorio ciudadano, centrándose en las acciones de colaboración entre las personas con las comunidades en la búsqueda de soluciones a problemas reales. El investigador participa activamente en el proceso, sin alejarse del contexto, obteniéndose un beneficio esperado en la investigación y los conocimientos para la comunidad, los cuales se pueden aplicar de inmediato.

Por otra parte, está la Investigación-creación, en el modelo propuesto por Findeli, Brouillet, Martin, Moineau y Tarrago (2008, citados en Delgado, Beltrán, Ballesteros \& Salcedo, 2015), en donde se exponen dos consideraciones que permiten entender la investigación en el proceso creativo:

La primera, denominada multidisciplinar, cuya meta es la producción de conocimiento, consiste en la conceptualización de una problemática compartida y conocida por todas las disciplinas que han acordado trabajar un proyecto determinado; esto implica una integración de las diferentes visiones que cada disciplina tenga del fenómeno que se quiere abordar y no una mera yuxtaposición de diferentes puntos de vista. La segunda dimensión es denominada transdisciplinar y consiste en que el conocimiento entregado por las disciplinas analíticas debe ser "trans-formado" para que pueda concretarse en una acción, de manera que el conocimiento sea relevante para el usuario final, y no solamente para la comunidad científica.

A partir de estas metodologías, los procesos de inmersión en diferentes comunidades del Departamento de Nariño, con ejercicios de empatía y el análisis de las investigaciones realizadas a través de los procesos ejecutados por el Centro de Innovación Social, se ha descubierto que "hay un gran número de personas que trabajan en procesos sociales con componentes innovadores y de empoderamiento comunitario que no llegan a ser concluidos porque van perdiendo valor en su desarrollo". Dicho de otra manera, son experiencias propias de líderes comunitarios que se dejan de lado al no adquirir valor ni la importancia que se les debería dar. Terminan, entonces, por ser sólo prácticas irrelevantes en el territorio, pero que realmente ya son un trabajo adelantado por la misma comunidad para resolver algunas problemáticas que se suscitan en sus contextos diarios, usando sus propios recursos, su propio ingenio y creatividad para hacer lo que pueden. Ese necesario valor social de empoderamiento y apropiación de conocimientos se podría 
generar a través de estrategias metodológicas, como son los laboratorios ciudadanos contextualizados en la cultura y el territorio. De esa manera, se fomentan las interacciones con personas que puedan ser expertas en determinados temas relacionados con el quehacer rural y puedan fortalecer, potenciar y acelerar esos procesos mediante el intercambio de saberes, la experimentación y el prototipado. Además, se visibilizan procesos innovadores para ser promovidos y replicados, buscando que estos proyectos sean conocidos por más personas y puedan implementarlos en sus propios territorios.

\section{Grupos de Interés}

Líderes comunitarios, familias campesinas, promotores de procesos sociales y asociaciones comunitarias son quienes vivencian y evidencian las problemáticas del territorio en su contexto cotidiano, y quienes deberían llevar a cabo programas, planes o proyectos que permitan el empoderamiento comunitario mediante estrategias metodológicas de trabajo colaborativo y co-creación. Se generan así respuestas alternativas a dichas problemáticas.

Como estudio de caso se propuso a una asociación campesina comunitaria denominada Asociación de Cafeteros de la Vereda El Páramo del Municipio de Taminango (ASOCAMPAT), al norte de Nariño. Ésta tiene como su centro de trabajo una finca que se ha convertido en el imaginario de la comunidad de la vereda como un lugar de desarrollo. A pesar de ser privada, su propietario, don Héctor Erazo, ha permitido que todo aquel que quiera recuperar la dignidad de ser campesino pueda usar el espacio para desarrollar procesos comunitarios y ser bienvenido. Además, se le brinda el apoyo necesario para que aprenda métodos y técnicas para vivir bonito, como dice el mismo señor.

En aquel lugar y por iniciativa de su líder se pretendió recuperar las costumbres campesinas y darle valor a su ser rural. A través de la siembra de café han llevado a cabo procesos de innovación en cuanto a prácticas sostenibles y limpias, para poder producir un café especial de alta calidad. El lugar cuenta con un laboratorio de catación de café, procesos de purificación de aguas residuales del ejercicio cafetero. Por lo tanto, era necesario, en voz de sus propios habitantes, que los procesos sean fortalecidos, ya que ellos no tienen muchas posibilidades de salir a los centros urbanos y asistir a la academia especializada, aunque sí una completa disposición de aprender nuevas técnicas y confrontar sus saberes para fortalecer y potenciar las prácticas que tal vez les ha llevado mucho tiempo descubrir. Con la ayuda de estas interacciones redescubrieron cómo acelerar los procesos y generar nuevas habilidades para solventar sus problemáticas, mediante respuestas alternativas en camino hacia un vivir bonito. 


\section{Empatía e Inmersión}

Es verdad que el Departamento de Nariño, culturalmente cuenta con ejercicios de participación comunitaria como las mingas, en donde todos se unen para colaborar en la construcción de bienestar social, no sólo en infraestructura sino, también, en el cuidado de los habitantes comunes y del pensamiento. Precisamente, es de suma importancia recuperar esas actividades culturales y esos imaginarios colectivos para potenciarlos hacia el empoderamiento y el desarrollo integral de las comunidades, sin tener que depender exclusivamente de las instituciones y sus recursos.

En primera instancia, para reducir las barreras de trabajo con la comunidad de dicha asociación, se realizó una actividad previa al proceso creativo denominada fase de empatía (taller realizado en la comunidad como inmersión y auto-reconocimiento). Este encuentro se llevó a cabo a través de ejercicios didácticos basados en la metodología de innovación social FEELING, en donde las personas se auto-reconocen como actores principales dentro del proceso y reconocen sus habilidades como principal aporte a la construcción colectiva $\mathrm{y}$ al trabajo colaborativo.

Resultados del taller:

- Se definieron sistemas a través de la experimentación a prueba y error se aprende, generando procesos de innovación que les ha llevado muchos años de experiencia.

- Los recursos que utilizaron son propios, los conocimientos son transferidos entre generaciones, trayendo consigo varias evoluciones. Después de tener éxito con la experimentación se celebró y se compartió con la comunidad.

- El quehacer y el trabajo diario fueron parte del tiempo de ocio, pues su trabajo los motivó a seguir adelante y progresar como asociación y familia.

- La creatividad hizo parte de la disciplina y desde ahí se implementó el saber.

- La idea de dibujar y colorear nuevamente resultó un poco difícil, porque les asustó expresar sus conocimientos a través de dibujitos, pero los motivó a intentar resumir todo lo que pensaban en unos pequeños trazos.

- Después de la guía de súper-poderes que realizaron, todos quedaron con poderes (habilidades) que no conocían, lo cual quieren seguir utilizando para ser grandes súper héroes y cambiar poco a poco su comunidad.

- Durante el desarrollo del taller muchos se encontraban felices y motivados.

- Ellos expresaron que es mejor prestar atención a cada detalle de la experimentación y que luego de especializarse en el conocimiento podrán realizar una réplica.

Una vez superadas las barreras y creados lazos de afecto, se hizo un levantamiento de la línea base y el estado actual de la comunidad, caracterizando sus condiciones socioculturales, de trabajo comunitario y, principalmente, de percepción de la innovación social como herramienta para el desarrollo de procesos colaborativos. Así se tuvo una perspectiva inicial, sobre la cual realizar las mediciones y determinar los indicadores de impacto a corto plazo, descubriendo los siguientes efectos: 
- Las personas fueron conscientes de que los procesos políticos y administrativos de las instituciones han convertido a la ciudadanía en agentes pasivos, que solo son receptores, en muchos casos indiferentes e insatisfechos de los proyectos que se ejecutan en sus territorios.

- Fue muy importante la presencia de representantes y líderes de grupos comunitarios, puesto que a partir de su actuar en la comunidad se han generado nuevos procesos sociales. Los participantes expresaron la necesidad de que se valide y fortalezca tanto a líderes como a sus procesos, mediante nuevas metodologías y herramientas que ayuden a generar cambios y, sobre todo, a activar la participación comunitaria.

- Desde la ciudadanía existe la necesidad de realizar procesos de empoderamiento comunitario, fomentando una cultura que se proyecte al cambio, con nuevos métodos y nuevas formas de pensar, manteniendo las raíces y recuperando esos saberes ancestrales del territorio. Es ahí donde cobra pertinencia la implementación de proyectos creados desde la base, a través de estrategias de innovación social que se ejecuten en las comunidades buscando un bienestar integral de la sociedad.

- Siempre es de gran importancia dar valor a la participación y a la toma de decisiones en comunidad, generando estrategias de encuentro e interacción entre los habitantes del territorio y agentes externos con actividades culturales y de activación comunitaria, que fortalezcan sus habilidades, capacidades creativas y propositivas. De este modo, la comunidad colabora, propone, experimenta y gestiona a través de sus propios medios alternativas de solución a problemáticas que ella misma es capaz de focalizar.

\section{Propuesta de Empoderamiento Comunitario}

El empoderamiento es un concepto relevante y transversal en el proceso de desarrollo social sostenible; es una traducción literal de la palabra inglesa empowerment, término que ha ganado importancia a partir de la década de los ochenta en temas relacionados con las luchas feministas, especialmente en la Conferencia Mundial de las Mujeres de Naciones Unidas, realizada, una en Nairobi (1985) y otra en Beijing (1995).

A este respecto, Foucault (1999) afirma que el empoderamiento va más allá de la acción del poder y se suscita, más bien, en los procesos de creación y fortalecimiento de las condiciones propias de un individuo o de un colectivo en sus contextos materiales y subjetivos, incentivando su participación y la toma de decisiones. Así mismo, en el control, uso y acceso a los recursos para crear un entorno de bienestar.

El empoderamiento comunitario rural, entonces, implica la gestión de herramientas para el desarrollo de proyectos comunitarios y colaborativos mediante procesos de innovación social. Por consiguiente, requiere potenciar y fortalecer las habilidades comunitarias para consolidar un movimiento social más efectivo, apoyado en la autodeterminación 
de las personas, buscando su propio desarrollo y bienestar claramente articulado con las instituciones y agentes que hagan frente a la exclusión social, la estigmatización, la discriminación y la vulneración de los derechos humanos.

Es así, como el configurar una estrategia metodológica genérica para el desarrollo de procesos de innovación social en las comunidades, anclados a procesos reales y contextualizados en el territorio, bajo el concepto de laboratorio ciudadano y mediante herramientas didácticas, permitió lograr el empoderamiento comunitario y una efectiva apropiación de conocimientos en la interacción con personas externas. Para llevar a cabo dichos procesos paso a paso, liderados por representantes de las comunidades, se buscó también la apropiación práctica de estas estrategias desde sus saberes, quehaceres y recursos, con sus capacidades y habilidades, generando puntos de desarrollo y la construcción de tejido social alrededor de un buen vivir.

Las mesas de trabajo para la construcción de la política pública de innovación social, permitieron reconocer este deseo en los líderes del Departamento, puesto que su sentir siempre fue el poder contar con herramientas o mecanismos que les ayuden a realizar su proceso en comunidad y buscar de una manera efectiva la vinculación de otros actores para trabajar alli en su territorio donde se suscitan las problemáticas.

\section{Investigación y Desarrollo de la Estrategia Metodológica}

Una estrategia metodológica para el desarrollo de procesos colaborativos de innovación social en entornos rurales, fortalece las capacidades y habilidades creativas de la ciudadanía para afrontar problemáticas en comunidad. Esto se logra con la creación de respuestas alternativas en sus contextos cotidianos, fomentando el empoderamiento comunitario frente a su propio desarrollo. Para ello, es importante la apropiación del conocimiento, el co-diseño, la adaptación de tecnologías a bajo costo y el desarrollo de prototipos. Todo esto bajo una cultura libre que facilite la intervención de comunidades específicas con potencial de trabajo comunitario, las cuales posteriormente sean promotoras y replicantes de esos procesos, apoyando así a nuevas asociaciones comunitarias y a nuevos líderes.

Es así como la primera fase del proceso cubrió la investigación acerca de los laboratorios ciudadanos y sus distintas configuraciones para determinar las características relevantes y aplicables en un proyecto de diseño. Así se estructuró una nueva estrategia metodológica traducida en un artefacto tipo libro objeto (manual de herramientas creativas y experiencias de uso), que permitió a las comunidades desarrollar procesos de innovación social en el territorio de una manera eficiente, organizada y práctica, con recursos que tenían a la mano. De este modo se sirvieron de las tecnologías sociales, digitales y de comunicación para desarrollar, en conjunto con colaboradores, respuestas alternativas a problemáticas que habían focalizado en sus territorios. 
En la segunda fase se realizó el testeo e implementación de esta estrategia y de su caja de herramientas con una comunidad como caso de estudio, para lo cual se abrió una convocatoria a colaboradores y voluntarios que estén dentro de unos roles específicos, con capacidades y habilidades determinadas bajo unos perfiles requeridos para abordar las problemáticas que la comunidad designe como ejercicio de pilotaje. Fueron ellos quienes aportaron con sus capacidades a la co-creacion de un prototipo como respuesta alternativa a dicha problemática, todo esto bajo un modelo de laboratorio ciudadano realizado en el contexto.

Setrató, pues, de una residencia de trabajo en el territorio dondesesuscitan las problemáticas y en donde la comunidad aportó con estadía y manutención a los colaboradores y estos, a su vez, con sus conocimientos, experticia, tiempo y energía para desarrollar dicho proceso de innovación social por medio de la estrategia metodológica que se propuso.

\begin{tabular}{|c|c|c|c|c|}
\hline Imput & Actividades & Output & Outcomes & impacto \\
\hline $\begin{array}{l}\text { - Integrantes asociación } \\
\text { ASOCAMPAT } \\
\text { - Lideres comunitarios -. } \\
\text { Colaboradores y voluntarios } \\
\text { - Finca Asocampat en el } \\
\text { páramo de Taminango } \\
\text { - Aliados estratégicos } \\
\text { CISNA Y fundación social } \\
\text { - Recursos logisticos. } \\
\text { - Transportes. } \\
\text { - Material de trabajo } \\
\text { didactico. } \\
\text { - toolkit, herramientas } \\
\text { metodológicas. } \\
\text { - Material de prototipado }\end{array}$ & $\begin{array}{l}\text { - Mapeo y selección de } \\
\text { problemáticas por parte de } \\
\text { la comunidad. } \\
\text { - Jornada de creación de } \\
\text { retos de trabajo } \\
\text { - Jornada de autogestión } \\
\text { de recursos } \\
\text { - jornada de ideación, } \\
\text { experimentación y co- } \\
\text { diseflo } \\
\text { - construcción, testeo e } \\
\text { implementación del } \\
\text { prototipo }\end{array}$ & $\begin{array}{l}\text { - Mapa de problemas } \\
\text { construido } \\
\text { colaborativamente } \\
\text { - Fichas de caracterización } \\
\text { de las problemáticas } \\
\text { seleccionadas. } \\
\text { - Gulas con Retos de diseño } \\
\text { propuesto en comunidad. } \\
\text {-Fichas de } \\
\text { autorreconocimiento de } \\
\text { capacidades y habilidades de } \\
\text { las personas. } \\
\text { - Ideas y propuestas pro } \\
\text { grupos de trabajo } \\
\text { - Prototipos construidos y } \\
\text { testeados por grupo de } \\
\text { trabajo } \\
\text { - Implementación de } \\
\text { prototipo funcional }\end{array}$ & $\begin{array}{l}\text { - Participación de la } \\
\text { comunidad frente al } \\
\text { emprendimiento de } \\
\text { procesos de innovación } \\
\text { social. } \\
\text { - Propuestas alternativas } \\
\text { de solución a partir de } \\
\text { retos de trabajo } \\
\text { colaborativo e inteligencia } \\
\text { colectiva } \\
\text { - Personas con } \\
\text { capacidades y habilidades } \\
\text { para desarrollar tareas } \\
\text { comunitarias. } \\
\text { - Desarrollo de actividades } \\
\text { comunitarias en pro de } \\
\text { nuevos proyectos sociales }\end{array}$ & $\begin{array}{l}\text { Empoderamiento comunitario } \\
\text { fortalecimiento capacidades y } \\
\text { habilidades, creativas y de } \\
\text { recursividad. } \\
\text { apropiación del conocimiento } \\
\text { en co-diseño } \\
\text { Capacidad de afrontar } \\
\text { problemas en comunidad. } \\
\text { Capacidad de trabajo } \\
\text { colaborativo }\end{array}$ \\
\hline
\end{tabular}

Tabla 1. Testeo e implementación de estrategia

\section{¿Qué es un Laboratorio Ciudadano?}

Setrata de un espacio de creación colaborativa que propicia el empoderamiento social y territorial, donde la comunidad identifica y propone respuestas alternativas a problemáticas o necesidades sociales no satisfechas de su entorno, a través de la co-creacion con equipos multidisciplinares, en temas relacionados a retos definidos por la ciudadanía, generando un ambiente para el intercambio de saberes, experimentación y producción de prototipos como un factor decisivo en la construcción social. "Si en los laboratorios ciudadanos no se hace un esfuerzo también de aterrizar las soluciones, de prepararlas para acelerar su traslado a la realidad, vamos a convertirlos en espacios aislados y sin incidencia en las políticas públicas" (Rey, 2018). 


\section{El Comúnlab Rural: Laboratorio para Transformar Comunidades}

Es una nueva estrategia metodológica gestada a partir de la teoría de laboratorios ciudadanos con algunas adaptaciones que responden a las necesidades de las comunidades en contexto y se convierte en una propuesta para desarrollo de procesos de innovación social en entornos rurales, articulando a las distintas instituciones para fortalecer iniciativas comunitarias, para derribar esas barreras entre la ciudadanía y la institucionalidad. Se configura, entonces, como un nuevo ejercicio de creación colaborativa donde se fortalece el empoderamiento social y territorial. Estratégicamente diferenciado de los laboratorios ciudadanos tradicionales, principalmente en su etapa previa y, sobre todo, en el tema de gestión y relación entre organizadores, participantes y comunidad. Busca fluir en una dinámica cotidiana y está atento a las reacciones, cambios o necesidades que puedan suscitarse antes, durante y posteriormente a su aplicación in situ.

Esta metodología plantea, principalmente, estrechar las brechas sociales y establecer un espacio de encuentro temporal y físico para el trabajo colectivo, en el que durante varios días las comunidades afectadas abren sus puertas a los colaboradores y voluntarios que son personas con conocimientos diversos y comparten el interés de resolver desafíos puntuales identificados por las mismas comunidades. Se trabaja para generar círculos de convivencia que trascienden los espacios de creación e investigación del laboratorio ciudadano común.

El actual laboratorio se definió por la colaboración, la participación, el cuidado de los comunes, una perspectiva sostenible y de conocimiento libre. Todo esto posibilitó generar procesos dinámicos donde el intercambio de saberes, la experimentación y la producción de prototipos se enfocó en brindar alternativas de solución a problemas esenciales con la participación de colaboradores de la misma comunidad y externos, de diversas disciplinas y con múltiples conocimientos, que aportaron y brindaron una amplia gama de perspectivas para generar distintas y nuevas alternativas a los problemas. Para dicho propósito se estructuró, dentro de la metodología, una serie de momentos que son esencialmente una forma de llevar a cabo acciones conducidas en una lógica organizacional, que facilitó el desarrollo del proceso de manera alternativa, no rígida pero sí continuada y que proporcionó hitos o pequeñas victorias en cada etapa. De este modo, se mantuvo activo el proceso y la energía de sus participantes de principio a fin, al entender que el propósito primordial era el proceso en sí mismo, como el ejercicio de empoderamiento y apropiación de saberes por parte de la comunidad, y que no finalizó con la fabricación del prototipo, sino que fue su implementación la forma de validar dicho proceso. Se puso, así, un punto de partida a la autogestión de una comunidad para su propia transformación. 


\section{Estrategia Metodológica}

\section{- Etapa 1: Confiar}

En principio se hizo necesario un ejercicio de acupuntura social en el territorio, determinado por la inmersión y el acercamiento a las comunidades mediante actividades participativas de activación ciudadana. Fueron momentos dinámicos y didácticos que rompieron barreras institucionales y generaron lazos de afecto: talleres o dinámicas culturales en los que lo importante fue darle a la comunidad la oportunidad de expresarse y, de paso, recopilar información que focalizó ejes territoriales, líderes que podían ser un punto de partida y detonantes de empoderamiento comunitario.

\section{- Etapa 2: Sentir}

Se partió de la pregunta: ¿Dónde sientes el problema? Esta etapa trató la identificación de oportunidades de acción. Una vez determinadas, fue necesario realizar una serie de actividades que permitieron a las personas de la comunidad, convocadas abiertamente, purgar sus necesidades y encontrar una problemática general anclada a un proceso real de su diario vivir, respondiendo así a una deseabilidad en común.

- Fase 1: mapeo de problemáticas, denominado Mapatón, para el kit de herramientas. Fue un ejercicio en el que las personas dibujaron o proyectaron una imagen de su territorio para identificar, situar y priorizar una serie de problemáticas, en común acuerdo. Se determinó como hito principal la formulación de un reto, siendo éste el eje que atraviesa todo el proceso.

- Fase 2: en la cual la comunidad aportó ideas base y genéricas a partir de su experiencia, para darle apertura a la convocatoria de proyectos, enfocándose en alternativas novedosas y viables de solución a los retos propuestos. Así, desde el inicio se le dio valor a la opinión comunitaria y al imaginario colectivo territorial.

\section{- Etapa 3: Nacer}

¿Quiénes gestamos? Bajo esta pregunta se busca conformar una red de trabajo y para eso fue necesario articular el proceso entre líderes, comunidad, organización y la institucionalidad para generar una fuerza de gestión y trabajo alrededor de la gobernanza del proceso. Siempre es pertinente tener una base de actores y gestores para que el proyecto sea viable, sostenible y posteriormente replicado.

- Fase 1: a partir de aquí se hizo la apertura del proceso con una convocatoria inicial de ideas de proyectos. Ésta se debió dar a partir de la institucionalidad, que sirvió como aval y soporte del proceso, además de hacer también la posterior preselección de los proyectos que cumplieran con las características de la convocatoria y tuvieran 
los mejores aportes de innovación y desarrollo, enfocándose en buscar respuestas alternativas de solución a los retos propuestos.

- Fase 2: Hackathon y selección. En esta etapa se seleccionó, junto con la comunidad, las mejores propuestas de ideas de proyecto para cada una de las problemáticas (máximo tres ideas por la viabilidad de realización del laboratorio). Se citó a cada uno de los postulantes de los proyectos preseleccionados para una jornada compartida de trabajo tipo hackathon presencial o virtual. En esta jornada se hizo crecer la idea enfocándola al contexto y al final se realizó un prototipo visual de la idea mejorada, se expuso a la comunidad y mediante votación se eligió los proyectos que se realizarían en su territorio.

- Fase 3: una vez seleccionados los proponentes, se hizo con ellos una visita al territorio y junto con la comunidad se realizó una investigación más exhaustiva de las problemáticas priorizadas. Con herramientas de investigación etnográfica de inmersión y situacional, se construyó un documento con información relevante y de primera mano que sirvió para el desarrollo posterior de los proyectos.

\section{- Etapa 4: Colaborar}

Abrir el proceso a colaboración libre y voluntaria de cualquier sector. Una vez seleccionados los proyectos que se llevarán a cabo, se abrió una convocatoria de colaboradores con perfiles acordados entre los postulantes seleccionados y líderes comunitarios, dentro de cada proyecto deberá haber mínimo cuatro voluntarios aparte del postulante del proyecto. Los voluntarios pueden ser externos o de la misma comunidad y, adicionalmente, debe haber una persona o dos de la comunidad que conozcan en detalle la problemática que el proyecto va a afrontar.

Dentro de estos perfiles, se sugirieron cuatro tipos de roles:

- Maker: persona con habilidades y conocimientos de fabricación, que apoya en la proyección, co-diseño y construcción de los prototipos, no necesariamente objetuales o en la fabricación de artefactos, sino de prototipos tipo servicios, metódicos, gráficos o textuales.

- Conocedor: persona con conocimientos técnicos o académicos pertinentes a la problemática priorizada, que aporta desde su experticia en el desarrollo de las propuestas de solución al reto planteado.

- Mediador social: persona con habilidades de mediación entre las comunidades y grupos de trabajo, propiciando momentos de encuentro e interacción para el fortalecimiento de la gestión y gobernanza del proyecto.

- Documentador audiovisual: persona con capacidades y habilidades para la creación de contenidos comunicativos, gráficos y audiovisuales que ayuden a promocionar y visibilizar tanto el proceso como los proyectos y también la creación de manuales para la posterior réplica. 
El papel del colaborador en el proceso de ejecución del laboratorio fue determinante para el desarrollo de las propuestas seleccionadas. Su energía y disposición de trabajo en equipo permitieron abordar las problemáticas desde distintas perspectivas para enriquecer los proyectos y llevarlos a buen término en un contexto de innovación. La relación de trabajo en el laboratorio debe ser horizontal entre mentores, promotores, colaboradores, mediadores y comunidad en general. Esto supone una oportunidad para el intercambio de conocimiento, el aprendizaje teórico-práctico y la interacción socio cultural. Más allá del aporte del tiempo laboral, lo que se buscó es que los participantes en el laboratorio y la comunidad en general tejieran redes de afectos y promovieran el bienestar común.

\section{- Etapa 5: Vivir}

Trabajo horizontal, abierto y libre in situ, donde se presentan las problemáticas. Es aquí donde se desarrolló el laboratorio. Los integrantes de cada equipo, líderes comunitarios en el rol de mediadores, representantes de la organización de apoyo, representantes comunitarios y, también, colaboradores en general, estuvieron enfocados en la búsqueda de la respuesta al reto, al tomar como base la idea del proponente, experimentando, cocreando y construyendo un prototipo funcional para dicho propósito. Durante el laboratorio hubo expertos invitados que brindaron asesoría en trabajo con comunidades, prototipado y conocimientos específicos para apoyar al desarrollo del proyecto. Además, se realizaron una serie de encuentros extra laboratorio que permitieron afianzar el tejido social y dinamizaron a la comunidad en torno a las temáticas relacionadas con los proyectos que buscan el bienestar común. Así, de voz en voz, se fortaleció la participación de la comunidad, como, también, su apropiación dentro de dicho proceso.

Durante 8 a 10 días de trabajo, en el laboratorio se plantearon cuatro momentos, para que el proyecto pudiera llegar a un punto satisfactorio para todas las partes al finalizar el tiempo del encuentro:

- El primer momento fue la empatía, presentación y contextualización: jornada de encuentro para conocerse, reconocerse y generar lazos de afecto; aquí se expusieron los resultados de la investigación en la primera etapa y los acuerdos de trabajo y normas de convivencia.

- El segundo momento fue el planteamiento y desarrollo de la propuesta, como también la asignación de tareas por roles y habilidades, y la gestión de materiales; esta fase terminó con la elaboración de un prototipo inicial. Se elaboró un listado de materiales necesarios para un prototipo funcional, los cuales se consiguieron con un dinero base que brindó la organización por medio de autogestión con la comunidad.

- El tercer momento fue la construcción, testeo, análisis y ajustes al prototipo final para generar una propuesta de acción estratégica. 
- El cuarto momento fue transversal a todo el proceso. Hubo una autoevaluación constante y socializaciones abiertas del proyecto, así como avances para fortalecer las ideas, los prototipos y las propuestas; la gestión de recursos necesarios dentro de la comunidad, también fue un proceso constante.

\section{- Etapa 6: Transformar}

Implementación y demostración del prototipo funcional. El laboratorio finalizó con una actividad de implementación abierta a la ciudadanía para dar a conocer el resultado y la acción planteada, con una puesta en marcha en los puntos focalizados por cada uno de los grupos. Así, la comunidad pudo ver los resultados en funcionamiento y el beneficio real. También se convirtió en una jornada de encuentro entre todas las personas involucradas en los proyectos y representantes de entidades que puedan prestar cualquier tipo de apoyo para la replicabilidad y sostenibilidad del proyecto.

\section{- Etapa 7: Impactar y proyectar}

Replicar procesos, publicar resultados bajo licencia libre y tejer red alrededor del proyecto. En esta etapa se realizó un encuentro entre todas las personas involucradas en los proyectos y representantes de entidades que puedan prestar apoyo a la replicabilidad y sostenibilidad del proyecto. En este encuentro se hizo público un manual de replicabilidad y se empezó a tejer una red de apoyo alrededor de cada uno de los proyectos.

\section{Actores clave de empoderamiento comunitario y la gestión colaborativa}

- La institución que propició el espacio de encuentro y el desarrollo del proceso como una forma de acercamiento a las comunidades con un pilar de transformación y apalancamiento de proyectos

- La comunidad, que es el foco del proceso y se fortaleció mediante la toma de decisiones.

- Los colaboradores que dinamizaron el proceso y aportaron al fortalecimiento de habilidades y capacidades en comunidad.

\section{Consideraciones}

Los resultados obtenidos dentro del laboratorio, deberán ser publicados bajo licencias libres que reconozcan al autor, y se sugirió que se aplique la cláusula de compartir igual (en licencia de Creative Commons sería BY-SA). A su vez, siempre que se muestren dichos proyectos en festivales o exposiciones, y que se reproduzcan sus imágenes en catálogos o páginas web, deberá hacerse constar su relación con el Laboratorio de Innovación Ciudadana, ComúnLab. Asimismo, deberá hacerse constar siempre los nombres de las personas que han colaborado en el desarrollo de los proyectos. 


\section{Caja de Herramientas}

Determinantes:

- Es un artefacto analógico que podría ser proyectado posteriormente en el entorno virtual; pero, para efectos del desarrollo del proceso en los territorios, se hace necesario que sea un elemento físico a manera de libro objeto tipo manual de procedimientos.

- Contiene en su conjunto el compendio metodológico a través de elementos (guías o tarjetas), además de materiales didácticos con sus respectivas fichas de requerimientos y consejos para llevar paso a paso el laboratorio, de manera intuitiva y fácil de aplicar.

- Este artefacto es un libro de consulta, que puede ser usado por uno o varios actores clave para fomentar el empoderamiento comunitario y la gestión colaborativa.

- De fácil compresión, uso y adaptabilidad.

- Está dividido en etapas y fases para su ágil puesta en práctica.

- Su diseño y conceptualización le permite ser replicado, compartido y apropiado.

Requerimientos analógicos:

- Estructura modular

- Materiales resistentes para un uso constante

- De bajo coste y fabricación rápida

- Compacto y ligero para su fácil transporte

- Es un contenedor en sí mismo

\section{Resultados}

\section{- El ComunLab Rural}

El Laboratorio para transformar comunidades rurales se llevó a cabo en agosto de 2019 en la Vereda EI Páramo del Municipio de Taminango, Nariño. Para dar respuestas alternativas a los retos definidos por habitantes de la comunidad en un mapeo de problemáticas, dicho proceso contó con el apoyo estratégico de la Subsecretaria de Innovación, a través del Centro de Innovación Social de la Gobernación de Nariño (CISNA), en su Programa de laboratorios ciudadanos, y también con apoyo de la Maestría en Diseño para la Innovación Social (MADIS) de la Universidad de Nariño. Se convocó de manera abierta y participativa propuestas e ideas de proyectos a desarrollarse colaborativamente por equipos multidisciplinares e interculturales, de la mano con la comunidad y colectivos locales dentro del contexto cotidiano del territorio, contando siempre con el apoyo continuo de mentores especializados, especialistas técnicos, mediadores locales y la ciudadanía en general, para desarrollar, testear e implementar las soluciones prototipadas.

\section{- Retos}

En la jornada de mapeo con la comunidad y después de una rueda de pensamiento, se determinaron tres problemáticas a afrontar, que se convirtieron en los siguientes retos: 
1. ¿Cómo se podría recuperar la identidad cultural y la dignidad campesina para fortalecer el desarrollo y el bienestar social de los territorios rurales del Departamento de Nariño?

2. ¿Cómo se podría generar alternativas tecnológicas de bajo costo para la obtención, cuidado y movilidad del agua para uso agrícola y doméstico en entornos rurales de Nariño?

3. ¿Cómo se podría generar buenas prácticas ambientales y sostenibles, mediante recolección y tratamiento de basuras?

\section{Alternativas de solución propuestas}

Estas alternativas se recibieron mediante una convocatoria abierta y después de un filtrado que se llevó a cabo en conjunto con el equipo del CISNA, que ejerció en este proceso como aliado estratégico, y la comunidad de El Páramo, que seleccionó las propuestas que quería ver implementadas en su territorio.

Propuestas preseleccionadas:

Capacitar a campesinos en la elaboración de un sistema innovador que no requiere el uso de combustibles o energía eléctrica, mediante el uso de una bomba hidráulica de ariete para el acceso y uso de aguas cercanas a sus fincas; también está el buen uso de aguas residuales aprovechables dentro de sus hogares.

1. Eco-Taminango es una empresa asociativa de trabajo dedicada al reciclaje de elementos de cartón y de plástico en el Municipio de Taminango. La asociación surgió gracias a las necesidades y expectativas de la población de generar ingresos a personas discapacitadas del mismo municipio; posee una naturaleza jurídica en forma de organización no gubernamental sin ánimo de lucro. El lema fue: Un pacto con nuestro planeta tierra.

2. La pérdida de la identidad cultural y de dignidad campesina son una consecuencia del deterioro de las relaciones socio territoriales, el aumento de la brecha entre la población urbana y la rural, la pérdida de la biodiversidad y la explotación no sostenible de los recursos naturales. Esta problemática puede ser abordada desde una perspectiva multidimensional (social, política, económica y ambiental), al buscar, de manera integral, recuperar y fortalecer acciones de desarrollo territorial y bienestar social, garantes de dignidad, autonomía y soberanía de las poblaciones rurales.

3. Por tanto, es necesario desarrollar una serie de acciones que conlleven a los distintos actores y activos territoriales a la identificación de roles y recursos/capacidades, al fortalecimiento de la participación en espacios propios y exógenos, y a la disminución de brechas sociales. La consolidación de una interacción dinámica y sistémica entre la sociedad (medios de vida) y el territorio (recursos físicos), debe tener como objetivo impulsar iniciativas sostenibles de desarrollo local.

Por tanto, es necesario desarrollar una serie de acciones que conlleven a los distintos actores y activos territoriales a la identificación de roles y recursos/capacidades, al fortalecimiento de la participación en espacios propios y exógenos, y a la disminución de brechas sociales. La 
consolidación de una interacción dinámica y sistémica entre la sociedad (medios de vida) y el territorio (recursos físicos), debe tener como objetivo impulsar iniciativas sostenibles de desarrollo local.

Esta iniciativa consiste en generar Innovación Social Territorial (IST) a partir de la creación de una iniciativa piloto de Turismo Comunitario-Científico y de Recreación. Se proponen tres nodos:

A. Generación de información y de conocimiento: recolección de información multidimensional de la Vereda El Páramo a partir de fuentes primarias y secundarias (comunitarias, académicas, científicas e institucionales).

B. Identificación de Capacidades Territoriales, Turísticas y Científicas: reconocimiento de zonas de potencial turístico, científico y recreativo de la vereda EI Páramo. Reconocimiento a través de visitas in situ y en escenarios de articulación de actores territoriales.

C. Apropiación del conocimiento: uso del conocimiento generado en la iniciativa de innovación social para fomentar la cohesión social, la generación de ingresos y la sostenibilidad ambiental. Priorización participativa de alternativas de mejoramiento de calidad de vida individual y comunitaria.

Para la ejecución, se plantea contar con un experto temático, que aplique los enfoques diferenciales y de desarrollo; un grupo de makers, encargados de la aplicación de los instrumentos de recolección de información; un mediador social de la zona, como guía territorial y enlace comunitario; $y$ un documentador audiovisual, encargado del registro y la divulgación del proceso en plataformas digitales y otros medios de comunicación.

Como transformaciones territoriales (resultados), la Vereda El Páramo habrá participado de una iniciativa que mejorará la interacción entre sus integrantes, ya sean autoridades, líderes, lideresas, adultos mayores, jóvenes, niños y niñas. Esta identificará participativamente si su territorio posee potencial turístico, científico y recreativo, lo cual representaría una alternativa de desarrollo económico y social para la zona. La interacción comunidad-territorio plantearía una iniciativa de desarrollo local perfectamente replicable en otras veredas o municipios del Departamento de Nariño. La obtención y apropiación de conocimiento a partir del trabajo comunitario y articulado con los demás actores del territorio (academia, instituciones, administración local y sociedad civil) dentro del proceso, será la base para la toma de decisiones comunitarias previamente analizadas y concertadas. Esto representa para El Páramo y sus integrantes: trabajo en equipo, intercambio de saberes, auto-reconocimiento, visibilización de problemáticas individuales, familiares y comunitarias, sentido de pertenencia y resolución de conflictos.

1. Sin Abuso y con Re-uso, la mejor forma de cuidar el agua no es abandonar su uso y únicamente detener su abuso sino, también, fomentar su re-uso. La mayor parte del agua que se utiliza en las diferentes actividades humanas diarias y cotidianas es de un solo uso. Lo que implica un mayor agotamiento de este recurso. Por ejemplo, el agua que utilizamos para lavar los platos o las manos no se reutiliza y se pierde en su primer y único uso. Se propone reutilizar el agua con la que nos bañamos o duchamos diariamente como suministro para baños e inodoros. Estos son dos de los usos diarios y más cotidianos que se hacen del agua como recurso. Al aprovechar la incidencia lumínica de Taminango, 
mediante una pequeña motobomba solar se reabastece un tanque para suministro para inodoros. Una sencilla bandeja recolectora de agua se instala en las duchas de las casas. (Explicación muy general)

2. Crear una Semillero Eco-activistas que fortalezca las relaciones interpersonales entre la comunidad de la Vereda El Páramo, frente a una problemática común, como la seguridad y soberanía alimentaria y el cambio climático, para lo cual se trabajará en tres líneas estratégicas:

a. Formación de eco-activistas.

b. Planificación de procesos en pro de la soberanía y la seguridad alimentaria frente al escenario de cambio climático.

c. Fortalecer capacidad de ejecución y réplica de las iniciativas priorizadas.

\section{Propuestas seleccionadas:}

La selección de las propuestas que se desarrollaron en el laboratorio de innovación social, estuvo a cargo de representantes y líderes comunitarios del corregimiento El Páramo de Taminango, siendo alli en el territorio donde se construirán e implementarán las alternativas propuestas, encaminadas a resolver las problemáticas que la misma comunidad ha priorizado.

Reto 1 = propuesta 3: Iniciativa Piloto de Turismo Comunitario - Científico y/o de Recreación.

Reto 2 = Propuesta 4: Sin abuso y con re-uso es la mejor forma de cuidar el Agua.

Reto 3 = Propuesta 5: Semilleros Eco Activistas.

\section{Criterios de evaluación:}

1. La propuesta, sí o no, argumenta la optimización de los recursos a bajo costo.

2. En qué medida la propuesta responde con pertinencia a la problemática descrita en cuanto a la temática y retos expuestos en la convocatoria y planteados por la comunidad de la Vereda El Páramo del municipio de Taminango.

3. Cuán claros y precisos se expresan los elementos que le dan valor a su propuesta con relación a las necesidades y deseos de las personas del contexto territorial abordado, manteniendo una intencionalidad de cambio.

4. Grado de claridad, originalidad y creatividad de la propuesta de innovación social y que permita propiciar sensaciones, significaciones, experiencias estéticas, deseos y usos.

5. Grado de viabilidad técnica, articulándose con las habilidades, capacidades y tecnologías actuales disponibles en el territorio.

6. Qué tan fácilmente es apropiable y replicable por las mismas comunidades. 


\section{- Proyectos implementados}

Al finalizar el laboratorio se implementaron tres proyectos, que se describen a continuación:

Vivir el agua - sin abuso y con re-uso: al aprovechar la incidencia lumínica de Taminango, mediante una pequeña motobomba solar se reabastece un tanque para diversos usos. Se aprovecha de los sistemas de recolección existentes en la finca, como el reservorio y el filtro de agua natural que permite reutilizar el agua utilizada en actividades domésticas.

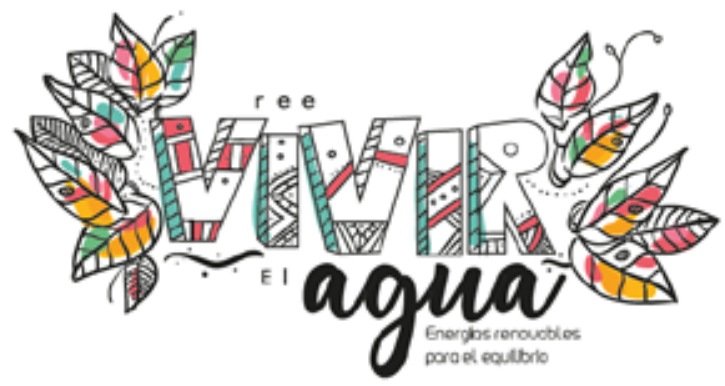

Figura 1. Proyecto Vivir el Agua

La estructura del panel es construida con materiales disponibles en la zona y su sistema eléctrico ha sido simplificado para que en su instalación no sea necesario tener conocimientos avanzados de electrónica. Este sistema les permite a las comunidades tener agua para riego en lugares donde antes no la tenían y en cualquier época del año. Colaboradores: Gabriel Tupaz, Andrea Estefanía Morales Mora, Rubén Eduardo Caipe Loaiza, Diego Barrera, Sairo Muñoz, Sneider Muñoz.

Vivir bonito: esta iniciativa consiste en generar innovación social territorial a partir de la creación de una iniciativa piloto de turismo comunitario en la Vereda El Páramo de Taminango, y la elaboración de un manual de replicabilidad con un paso a paso para que diferentes comunidades puedan emprender una iniciativa de turismo comunitario.

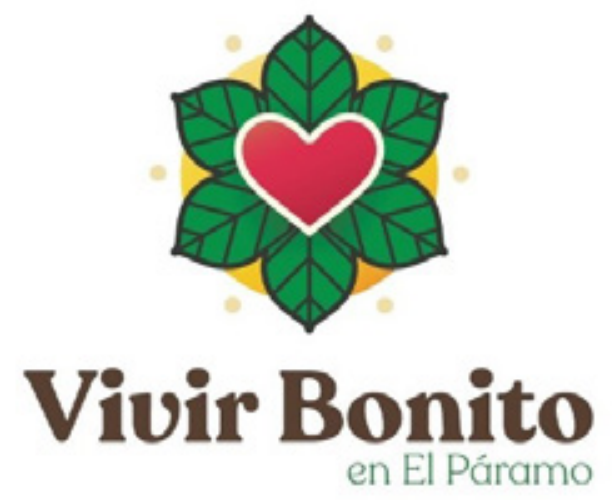

Figura 2. Proyecto Vivir Bonito 
El proyecto dejó diseñada una experiencia turística, un levantamiento de inventario turístico y un presupuesto para la experiencia Vivir Bonito en El Páramo. Colaboradores: Emmanuel Caicedo Guerra, Gloria Cheyenne Narváez Paz, Martín Reyes, María Camila Montenegro Erazo, Carolina Erazo, Leonardo Erazo, Luis Alfonso Riascos.

Semillero Ecoactivista: para fortalecer las relaciones interpersonales entre la comunidad de la Vereda El Páramo, frente a una problemática común como la seguridad y soberanía alimentaria y el cambio climático, se trabajó en tres líneas estratégicas: a) Formación de ecoactivistas; b) Planificación de procesos en pro de la soberanía y la seguridad alimentaria frente al escenario de cambio climático; c) Fortalecimiento de la capacidad de ejecución y réplica de las iniciativas priorizadas.

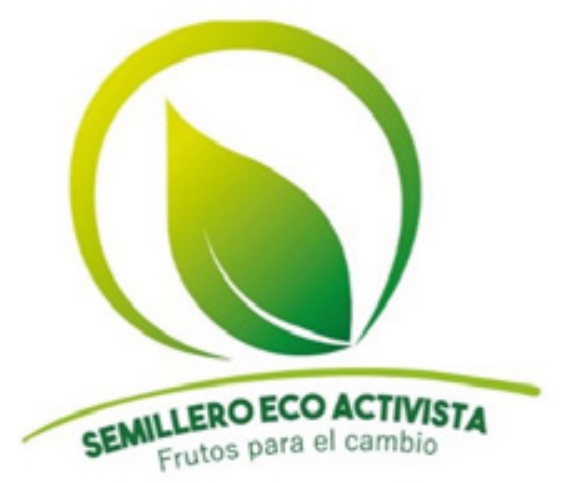

Figura 3. Proyecto Semillero Eco-activista

El proyecto dejó instalado un punto ecológico para la recolección de residuos sólidos, un taller para la transformación de residuos sólidos y un cronograma de actividades con estudiantes de la Institución Educativa El Páramo, así como un manual para replicar el punto ecológico. Colaboradores: Miguel bastidas, María Alejandra Narváez, Alejandra Rosas, Johana Paola Martínez Guerrero, Alejandra Bravo, Juliana Erazo, Mauricio Bravo

\section{Datos}

- 43 personas de la comunidad participantes en los proyectos desarrollados.

- 84 personas asistieron a la jornada de implementación final.

- Se vincularon directamente en los tres proyectos ocho niños, siete adultos, dos adultos mayores.

- Durante los días del laboratorio, se realizó una charla de un tema específico que se concretó con la comunidad previamente, de la misma forma hubo dos noches de cine al aire libre y una noche de cuentería.

- Así fue la agenda abierta del ComúnLab Rural:

Día 1. Cine al aire libre: El niño que domó el viento.

Día 2. Cuenteros Colectivo Galeras cuenta.

Día 3. Conversatorio: Ambiente Sostenible, por Alejandra Bravo y Johanna Martínez. Día 4. Conversatorio: Contabilidad básica, por Ángela Ponce. 
Día 5. Conversatorio: Manejo de herramientas TIC, por David Santacruz.

Día 6. Conversatorio: Asociatividad y asesoría legal, por Andrés Cuasapud.

Día 7. Cine al aire libre: Pequeña gran vida.

- 13 profesionales diferentes participaron en laboratorios, así: uno de Administración de empresas, uno de Sociología, tres de Diseño Industrial, uno de Ingeniería agroforestal, dos de Diseño gráfico, uno de Ingeniería industrial, uno de Técnico electromecánico, uno de Derecho y dos de Ingeniería ambiental.

- Los grupos de trabajo se conformaron por 13 colaboradores ajenos a la comunidad, 7 adultos y 8 niños integrantes de la comunidad de El Páramo.

- El laboratorio, durante los 9 días de trabajo, tuvo 320 visitantes, con un mayor porcentaje en el inicio y final del mismo.

- 18 personas capacitadas, no solamente de la comunidad de El Páramo sino, también, colaboradores que ampliaron su conocimiento en algún tema específico y ahora cuentan con la capacidad de generar réplicas y nuevo conocimiento.

\section{Limitación de responsabilidad}

- Cada grupo es responsable de administrar eficientemente los recursos monetarios asignados, además de gestionar nuevos recursos tanto técnicos como materiales y herramientas pertinentes al desarrollo del proyecto y prototipado. El acceso y uso de los recursos y medios técnicos aportados por la organización, fueron coordinados y supervisados por los responsables del laboratorio.

- Los organizadores no son responsables por los datos o los contenidos que utilicen los participantes. Así como tampoco lo son de los derechos de autor o aquellos derechos que correspondan a terceros conforme las leyes respectivas.

- El ComúnLab Rural es un evento que se desarrolla en varios días, por lo que confía en la responsabilidad de cada participante durante todo este período. La organización no se hará responsable de hurtos o extravíos de pertenencias personales, ni de daños físicos a las personas.

- El ComúnLab Rural no paga ninguna remuneración económica a sus participantes, al entender que es un espacio de colaboración y aporte de cada uno al bien de la comunidad.

- Colaboradores: personas con conocimientos, habilidades o capacidades técnicas, que aportan de manera libre y desinteresada con su tiempo de trabajo en el desarrollo de proyectos específicos de las comunidades, en un proceso continuo de aprendizaje e innovación para, así, dar apoyo a la ciudadanía en la creación de respuestas alternativas a necesidades comunes del contexto. Desempeñan un papel estratégico en la interacción con las comunidades y el entorno, para compartir saberes y generar apropiación social del conocimiento, al adoptar la misión de crear y mantener el valor social, y actuando siempre con respeto por el buen vivir, la igualdad y la equidad. 
- Voluntarios: son aquellas personas que poseen un carácter solidario y vocación de transformación social, que aportan de manera libre y desinteresada con su fuerza y energía de trabajo en el apoyo, acompañamiento o realización de tareas específicas dentro de un proyecto determinado, de acuerdo con su disposición laboral y temporal.

- Disposiciones legales: la Ley 720 de 2001 (Reglamentada por el Decreto nacional 4290 de 2005), por medio de la cual se reconoce, promueve y regula la acción, colaboración y voluntariado de los ciudadanos colombianos, define que: "Un colaborador o voluntario es toda persona natural que libre y responsablemente, sin recibir remuneración de carácter laboral, ofrece tiempo, trabajo y talento para la construcción del bien común en forma individual o colectiva, en organizaciones públicas o privadas, o fuera de ellas".

\section{Conclusiones y Lecciones Aprendidas}

Implementación de una herramienta que permite visualizar y localizar la cantidad de problemáticas u oportunidades de acción encontradas en el contexto, y cuáles de éstas se han abordado desde de iniciativas ciudadanas de innovación social, para especificar casos exitosos frente a casos desafortunados. Así, con las mismas personas se priorizó y enfocó una ruta de trabajo y unos ejes de acción, además de la cantidad de personas de la comunidad fuera de la asociación involucradas en estas actividades.

Rediseño de una alternativa metodológica de desarrollo de laboratorios ciudadanos enfocados en problemáticas de entornos rurales y con la participación directa de la comunidad en el proceso de experimentación y prototipado para la co-creación de soluciones contextualizadas al territorio, que favorezca el intercambio de saberes entre las comunidades rurales, permita la convergencia interdisciplinaria para ampliar discursos, debatir propuestas y construir conocimiento con respeto por las diferencias y así generar un proceso concertado y sostenible de desarrollo local.

El kit de herramientas para potenciar y fortalecer a las comunidades en el desarrollo de sus propios procesos de innovación, además de la apropiación social de conocimientos, para validar mediante la experimentación sus propios saberes y generar nuevas alternativas de solución a problemáticas cotidianas. Esto, a través de un mecanismo de interacción entre expertos y las comunidades en el contexto rural, gestionando los saberes en la práctica para la retroalimentación y el registro de actividades significativas que pueden ser potencialmente replicadas.

El desarrollo de un plan estratégico de trabajo colaborativo para la ejecución de actividades a partir del co-diseño mediante la adaptación, apropiación de ciencia y tecnologías de bajo costo. La creación de prototipos y su implementación en el contexto real permitirán posicionar la gestión del conocimiento y la innovación social como elementos centrales dentro de los procesos de desarrollo, para empoderar e impulsar a las familias campesinas a afrontar retos para que mejoren su modo de vida en el campo y conserven los recursos naturales. 
Generación de una estrategia de visibilización de las acciones ciudadanas, mediante la sistematización de buenas prácticas e indicadores de impacto, que apoyen la gestión de estrategias de desarrollo económico y social en los campos económico, social, político, cultural y ambiental.

La creación de documentos y manuales de replicabilidad, tanto de los procesos de empoderamiento como de los resultados de los laboratorios, permite la recuperación de saberes ancestrales a través de la realización de jornadas de inteligencia colectiva y trabajo colaborativo, frente a temáticas rurales, para generar un banco de conocimiento transferible y replicable.

\section{Referencias Bibliográficas}

Barroso, J. M. (2011). Políticas públicas e innovación social. Marcos conceptuales y efectos en la formulación de las políticas. Recuperado de http://www.academia.edu/865070/ Pol\%C3\%ADticas_p\%C3\%BAblicas_e_innovaci\%C3\%B3n_social._Marcos_ conceptuales_y_efectos_en_la_formulaci\%C3\%B3n_de_las_pol\%C3\%ADticas

Baskerville, R. (1999). La investigación de los sistemas de información con la investigaciónacción. Comunicaciones de la Asociación para Sistemas de Información 2 (artículo 19). Recuperado de http://cais.isworld.org

Congreso de la República de Colombia. (2001, 24 de diciembre). Ley 720 . Por medio de la cual se reconoce, promueve y regula la acción voluntaria de los ciudadanos colombianos (Reglamentada por el Decreto Nacional 4290 de 2005). Recuperado de https:// www.cerrejon.com/wp-content/uploads/2017/10/LEY-720-DE-2001-ACCIONVOLUNTARIA.-ilovepdf-compressed.pdf

Delgado, T.; Beltrán, E.; Ballesteros, M. \& Salcedo, J. (julio-diciembre, 2015). La InvestigaciónCreación como escenario de convergencia entre modos de generación de conocimiento. Iconofacto, 11(17). Recuperado de https://revistas.upb.edu.co/index. php/iconofacto/article/view/6356

Findeli, A.; Brouillet, D.; Martin, S.; Moineau, C. \& Tarrago, R. (mayo, 2008). Research through design and transdisciplinarity: A tentative contribution to the methodology of design research. En Simposio Focused-Current design research projects and methods. Swiss Design Network, Berna. Recuperado de https://swissdesignnetwork.ch/src/ publication/focused-current-design-research-projects-and-methods-2008/SDNPublication-2008_Focused.pdf

Foucault, M. (1999). Estrategias de poder (Ed. J. Varela \& F. Álvarez-Uría). Barcelona: Paidós. Recuperado de http://www.medicinayarte.com/img/foucault_estrategias_de_poder.pdf

Ferre Mora, M. (2015). Empoderamiento, participación y sentido de comunidad (Tesis de grado). Universitat Jaume I. Castellón de la Plana, España. Recuperado de http://repositori.uji. es/xmlui/bitstream/handle/10234/136547/ TFG_2014_ferreM. pdf?sequence=1

González, E. (1999). Sociología agraria y desarrollo local. Reflexiones a partir de estudios. La Habana: Universidad de La Habana.

Laverack, G. (2005). Using a domains approach to build community empowerment. Recuperado de http://www.oup.co.uk/journals/cdj/advance 
Laverack G. (2004). Health promotion practice: power and empowerment. London: Sage Publications. Recuperado de https://portal.findresearcher.sdu.dk/en/publications/ health-promotion-practice-power-amp-empowerment

Miranda, T. et al. (septiembre-diciembre, 2011) La Innovación y la transferencia de tecnologías en la Estación Experimental "Indio Hatuey": 50 años propiciando el desarrollo del sector rural cubano. Pastos y Forrajes, 34(4), 393-412.

Neira, A. (27 de enero de 2018). En 2017 aumentó el abismo entre millonarios y pobres. El Tiempo.

Rey, A. (2018). Post 576, Sobre los laboratorios ciudadanos en el sector público. Recuperado de: https://www.amaliorey.com/2018/06/30/sobre-los-laboratorios-ciudadanos-enel-sector-publico-post-576/

Ricaurte, P. \& Brussa, V. (mayo, 2017). Laboratorios ciudadanos, laboratorios comunes: repertorios para pensar la universidad y las Humanidades Digitales. Liinc em Revista, 13(1), 29-46.

Serra, A. (enero, 2010) Citilabs: ¿Qué pueden ser los laboratorios ciudadanos? La Factoría, la revista social. Recuperado de https://revistalafactoria.org/articulos/ citilabslaboratorios-ciudadanos

Sosa, I. (abril-junio, 2013). Empoderamiento comunitario en salud en tres municipios cubanos. Revista Cubana de Medicina General Integral, 29(2), 184-191. 